\title{
XIX.
}

Aus der Poliklinik des Herrn Dr. S. Goldflam in Warschau.

\section{Beitrag zur Aetiologie und Symptomatologie der Tabes dorsalis.}

$$
\text { Von }
$$

\section{Dr. A. Tumpowski.}

I. In vorliegender Arbeit habe ich mich bemüht, mit Berücksichtigung der Streitfragen bezüglich der Tabes dorsalis das umfangreiche Material von 257 Fällen zu ordnen und zu bearbeiten. Die Fälle sind theils aus der Poliklinik des Herrn Dr. S. Goldflam, theils aus dem mir guitigst von ihm zur Verfügung gestellten persönlichen Material entnommen. Zu diesen Streitfragen gehört vor allen Dingen die uber die Aetiologie der Tabes. Bekanntlich nehmen die meisten der gegenwärtigen Forscher an, dass die Lues das hauptsächlichste ätiologische Moment der Tabes abgiebt, einige sogar, wie Möbius und Oppenheim, versteigen sich bis zu der Behauptung, dass nur Diejenigen an Tabes erkranken können, welche vorher Lues überstanden haben. Diese Annahme wird hauptsächlich durch statistischanamnestische Daten begrtindet, welche fast einstimmig das sehr häufige Vorkommen der überstandenen Syphilis in der Anamnese der Tabiker aufweisen, was bekanntlich Fournier zum ersten Male veranlasste, der Syphilis eine eminente ätiologische Bedeutung beizulegen. Degegen sind klinische und anatomisch-pathologische Beweise für den Zusammenhang zwischen Syphilis und Tabes sehr spärlich, oft sogar sind sie dieser Hypothese, welche auch viele Gegner hat, geradezu widersprechend. $\mathrm{Zu}$ diesen Gegnern gehörte unter Anderen $\mathrm{Char}$ $\cot ^{1}$ ), welcher zwar der Lues gewissermaassen eine ätiologische Bedeutung zuschrieb, aber sie nur als nebensächliches Moment (agent provocateur) betrachtete und in erster Linie die erbliche Anlage betonte. Der bekannteste jetzige Gegner der obengenannten Hypo-

1) Leçons du Mardi à la Salpêtrière. Policlinique. 
these ist Leyden. Aus seiner Klinik ist auch die Arbeit Storbeck's ${ }^{1}$ ) hervorgegangen, in welcher die hauptsächlichsten Argumente contra zusammengefasst sind. Dieser Aufsatz ist so charakteristiseh, dass ich mir erlauben werde, das Wichtigste hier in Kurzem wiederzugeben. Seiner Meinung nach sind die Zahlen, welche von Syphilis bei Tabes dorsalis sprechen, sehr irreleitend, und ihre Grösse sehr oft von nebensächlichen Einflüssen abhängig. So z. B. bei Four nier, der bei 93 Proc. von Tabikern Syphilis gefunden hat, lässt es sich dadurch erklären, dass er als Syphilidolog meistens specifisch inficirte Kranke zur Behandlung bekam u. s. w. Auch davon ist die Grösse der Zahlen abhängig, dass man zu ihnen auch die zweifelhaften Fälle der Lues hinzurechnete. Ausserdem vergrössert sich im Anfangsstadium der Tabes der geschlechtliche Trieb, was wiederum die Vermehrung der Syphilis bei Tabikern hervorruft, die Syphilis ist jedoch bier nicht die Ursache der Tabes, sondern nur eine intercurrente Krankheit.

Noch eins muss man in Betracht ziehen, was die Grösse der Zahlen beeinflusst, nämlich, dass die allgemeine Ausbreitung der Syphilis unter Kranken (Nicht-Tabikern) ungefähr 25 Proc. beträgt, welche also immer abzurechnen sind. Dann ist es ja auch sehr schwer die überstandene Syphilis zu erkennen; es genügen dazu nicht die Aussagen der Kranken, nach welchen sich der Arzt sehr oft richtet, auch nicht dergleichen Residuen, wie vergrösserte Lymphdrüsen und Narben an den Geschlechtsorganen; die Härte des Schankers ist auch kein zuverlässiges Kriterium, weil sie, wie $\mathrm{Koch}$ behauptet, sehr oft das Resultat einer nicht entsprechenden Behandlung ist. Die öfters vorkommenden Aborte bei Frauen sind auch keineswegs Beweise für Lues. Die Syphilidologen führen auch Ziffern an, welche im Gegensatz mit der Ansicht über die ätiologische Bedeutung der Lues für Tabes stehen, so z. B. bat Lewin bei 800 syphilitischen Frauen kein einziges Mal Tabes gefunden, Reumont constatirte das Leiden bei 3600 Syphilitikern nur 40 mal, also 1,4 Proc. Tabes. ${ }^{2}$ ) Dies sind die wichtigsten Argumente, welche $\mathrm{S}$ torbeck in seinem Aufsatze gegen den Zusammenhang ron Syphilis und Tabes anführt. Die Gegner dieser Ansicht schöpfen ausserdem das Material zu ihrer Kritik aus der vollkommenen Unkenntniss der Art und Weise des Zusammenhanges zwischen diesen beiden Krankbeiten, - einer Unkenntniss, zu

1) Tabes dorsalis und Syphilis. Dissert.

2) Das hängt wohl damit zusammen, dass Tabes gewöhnlich Jahre lang nach der Ansteckung auftritt, die Syphilidologen dagegen haben es hauptsächlich mit Fällen in früheren Stadien zu thun. 
der sich selbst die entschiedensten Anbänger dieser Hypothese, wie Erb, Möbius, Oppenheim u. A., bekennen.

Die anatomisch-pathologischen Beweise für diese Behauptung sind sehr spärlich. Vor allen Dingen gehören hierzu Fälle von Tabes bei Eheleuten, die sich gegenseitig mit Lues angesteckt hatten ${ }^{1}$ ), dann das gleichzeitige Auftreten von Syphilis und Tabes bei demselben Individuum, ferner das Vorkommen der Ophthalmoplegia nuclearis ext. bei Tabikern, welche auch bei der Syphilis oft auftritt, endlich vereinzelte mikroskopische und Sectionsbefunde, welche bei einem and demselben Individuum neben tabetischen auch luetische Veränderungen bewiesen. ${ }^{2}$ )

Mit Berücksichtigung des oben Angeführten wenden wir uns jetzt an das uns zur Verfügung stehende Material. Ueberstandene Syphilis erkannten wir in folgenden Fällen: 1. im Falle der Arssage des Kranken, die sich auf die Diagnose des Arztes stützte; 2. beim Vorkommen eines Ulcus mit secundärem Exanthem; 3 . beim Vorhandensein eines Uleus von unbestimmtem Charakter, wenn zugleich andere speeifische Erseheinungen vorbanden waren, wie specifische Halsentzinndung, Plâques muqueuses u. s.w. Zweifelhafte, jedoch wahrscheinliche Syphilis diagnostieirten wir beim Vorhandensein eines zwar nicht sicher zu bestimmenden Ulcus, aber bei gleichzeitigem Vorkommen von Sterilität, häufigen Aborten und Gebären todter Kinder. Eine besondere Gruppe bilden Fälle mit weichem Schanker. ${ }^{3}$ )

1) Gold flam, Klinischer Beitrag zur Aetiologie der Tabes dorsalis. Deutsche Zeitschrift für Nerrenheilkunde. - Neuestens war auch in einem russischen medieinischen Blatt (Bibliotheka Wratscha. 1896. Mai) ein Fall von Marie und Bern ard angeführt: zwei Subjecte wurden gleichzeitig von derselben Frau im Jahre 1869 mit Lues angesteckt, im Jahre 1890 trat bei einem und 1891 beim anderen Tabes auf.

2) Bei 11 Sectionen fand Westphal nur 1 mal syphilitische Residuen bei Tabikern. West enh of fer fand bei 61 Sectionen von Tabikern zweifellose Syphilis in 24,6 Proc. und mit zweifelhaften Fällen zusammen in 44 Proc. M öbius (Schmidt's Jahrb. 1880. Bd. CLXXXVII) führt die Ansicht Virchow's über einen Sectionsfall an, dass die neben tabetischen gefundenen syphilitischen Veränderungen zweifellos die Ringe einer Kette sind. Um nicht aus dem Gebiete unseres rein klinischen Materiales herauszutreten, müssen wir den Leser in Bezug auf die anatomisch-pathologischen Fragen auf die Arbeiten von Leyden, Redlich, Obersteiner, Marinesco, Moxter u. s. w. verweisen.

3) Hirs chl in seiner Arbeit: „Die Aetiologie der progressiven Paralyse“ (Jahrb. für Psych. u. Neurolog. 1896. Bd. XIV. Heft 3) führt von den obigen ein wenig abweichende diagnostische Grundsätze an, und zwar hält er die Diagnose von überstandener Syphilis für gewiss, wenn 1. im Krankenhause die Sklerose und eine specifische Cur festgesetzt wurde; 2. wenn ein Ulcus mit secundärem Exanthem oder anderen allgemeinen syphilitischen Erscheinungen constatirt wurde; 3 . bei 
Das Resultat der Berechnung nach den eben angeführten Grundsätzen war folgendes: bei 257 Tabikern (darunter 31 Frauen) fanden wir

Sichere Syphilis . . . $100 \mathrm{mal}=38,9$ Proc.

Wabrscheinliche Syphilis . $\quad 51==19,8=$

Weicher Schanker ... $15==5,8=$

Andere anamnestische Daten, die irgend welche ätiologische Bedeutung haben können, sind:

Aborte und Sterilität ........ $12 \mathrm{mal}$

Geschlechtliche Ausschweifungen. . . . . $6=$

Alkoholische ........... $5=$

Erbliche Belastung ......... $4=$

Erkältung ............ $2=$

Im Puerperium ........... $1=$

Beschäftigung im Freien ....... $1=$

In den übrigen 54 Fällen konnten keine ätiologischen Momente aufgefunden werden. Wir mïssen noch hinzufügen, dass in der ersten Gruppe von Fällen neben der Syphilis auch andere krankheitserregende Momente vielleicht mitspielen, welche wir nach dem Muster von $\mathrm{Erb}^{1}$ ) hier gleich angeben:

Sichere Syphilis + Erblichkeit .........4 4 mal

+ weicher Schanker ...... $2=$

\pm Erkältung. .......... $2=$

+ geschlechtliche Ausschweifungen . $2=$

+ Alkoholmissbrauch ....... $1=$

$=\quad=\quad+$ Tripper + Erblichkeit ..... $1=$

Wahrscheinliche Lues + Erblichkeit . . . . . $2=$

$=+$ Trauma.......... $1=$

$=+$ Tripper + weicher Schanker $1=$

$=+$ Alkoholmissbrauch ..... $1=$

$=+$ Erkältung ........ $1=$

Wenn wir nun die Fälle von bestimmter Syphilis nebst anderen ätiologischen Factoren von der allgemeinen Zahl der sicheren Lues abziehen, so erhalten wir 88 Fälle, d. h. 34,2 Proc., in welchen die Syphilis allein in der Aetiologie figurirt. Die entsprechende Zahl in Erb's Statistik betrifft 27 Proc.

-nicht näher zu bestimmenden Krankheiten der Geschlechtsorgane, aber bei gleichzeitigem Vorhandensein von sich wiederholenden Aborten. Die Wabrscheinlichkeit von überstandener Lues nimmt Hirschl an 1. bei Ulcus in der Anamnese und Sterilität; 2. wenn der Kranke leugnet, irgend welche venerische Krankheit gehabt zu haben, und dabei typische Aborte vorgekommen sind. Würden wir die Grundsätze Hirschl's angewendet haben, würde der Procentsatz von Syphilitikern bei unseren Kranken etwas grösser ausfallen.

1) Zur Aetiologie der Tabes dorsalis. Berlin. klin. Wochenschr. 1891. Nr. 29. 
Was Franen anbetrifft, so sind bei ihnen folgende ätiologische Momente zu verzeichnen:

Ueberstandene Syphilis ......... 3 mal

Syphilis beim Mann und bei Kindern.. $2=$

Aborte und Sterilität ........12=

Syphilis + Erblichkeit ........ $1=$

Alkoholmissbranch ......... $1=$

Puerperium ............ $1=$

In 14 Fällen wurde keine Ursache constatirt.'

Wir sehen also, dass auch in unseren Fällen in ätiologischer Hinsicht die Lues die hervorragendste Rolle spielt, wenngleich nicht eine solche, dass andere krankbeitserregende Momente gänzlich auszuschliessen wären. Wenn wir sogar die Daten Erb's und Na e ge li's iaber die allgemeine Verbreitung der Syphilis ${ }^{1)}$, welche bis 25 Proc. betragen soll, bericksichtigen, so bleibt fast noch ein ebensolcher Procentsatz (wenn wir, wie es Storbeck thut, zu dem Procentsatz von bestimmter Syphilis die Hälfte von Fällen von wahrscheinlicher Syphilis hinzurechnen), den man schon ausschliesslich auf die specielle, wenn man sich so ausdrücken darf, tabeserzeugende Wirkung der Syphilis zurïckführen muss. Der von uns gefundene Procentsatz von Lues ist aber nicht so gross, dass wir uns der Ansicht von Mö bi us und 0 ppen hei m vollständig anzuschliessen vermögen. Auch die Gowers'sche Behauptung, dass man niemals bei einem Tabiker mit Bestimmtheit Syphilis ausschliessen kann, halten wir nicht für berechtigt; bis zu einem gewissen Grade könnte man mit eben solchem Recht behaupten, dass iuberhaupt bei Niemandem die Syphilis mit Sicherheit auszuschliessen sei. Diese Meinungen sind ebenso einseitig, wie die ganz entgegensetzte Ansicht Leyden's, welcher der Lues jede ätiologische Bedeutung abspricht und sie neben der erblichen Prädisposition, anstrengender Arbeit, Erkältung stellt. ${ }^{2}$ ) Dieser Umstand, dass wir in der Mehrzahl der Fälle, wo wir in der Anamnese keine venerischen Krankheiten finden, auch am häufigsten nicht im Stande sind, irgend ein anderes ätiologisches Moment zu finden, vergrössert noch den Werth der die Syphilis betreffenden Zahlen und bildet noch

1) Er b fand bei $\mathbf{5 5 0 0}$ krauken Nicht-Tabikern (progressive Paralytiker eingerechnet) die Syphilis in 22,5 Proc. Naegeli fand bei 1403 Tabikern Syphilis in 46 Proc. und bei 1450 Nicht-Tabikern nur in 9,5 Proc. der Fälle (citirt in Hirt's Pathol. und Therapie der Nervenkrankheiten).

2) Storbeck, l. c., Leyden, Die neuesten Untersuchungen über die pathologische Anatomie und Physiologie der Tabes (Zeitschrift für klin. Medicin. 1894. Bd. XXV). Eben dasselbe behanptet Berger auf Grund der Untersuchung von 185 Fällen von Tabes (Schmidt's Jahrb. 1879. Bd. CLXXXIII). 
eine Stütze mehr für die Behauptung über die eminente ätiologische Wichtigkeit der Syphilis für die Entstehung von Tabes dorsalis. Die endgiltige Entscheidung dieser ätiologischen Streitfrage verlangt die Feststellung zweier Punkte, nämlich, die präcise Diagnose der überstandenen Syphilis und die Kenntniss des Pathogenie dieses Leidens. Den durch Gowers, Möbius, Oppenheim, Strümpell u. A. ausgesprochenen Hypothesen über die Entstehung der Tabes unter der Wirkung eines specifischen, syphilitiscben Giftes kann man wohl keine besondere wissenschaftliche und praktische Bedeutung beilegen, da noch nichts über dieses Gift bekannt ist. Am vorsichtigsten geht in dieser Hinsicht Erb vor; er schreibt zwar der Syphilis in der grössten Mehrzahl (96-97 Proc.) der Fälle eine entschiedene ätiologische Bedeutung zu, hält sich jedoch von allen Erklärungen diese Zusammenhanges fern. ${ }^{1}$ )

Was die Zeit anbetrifft, in welcher nach der Ansteckung mit Syphilis die ersten Zeichen von Tabes auftraten, giebt uns die nachstehende Tabelle, welche 116 Fälle umfasst, einen Ueberblick:

\begin{tabular}{|c|c|c|c|c|}
\hline \multicolumn{3}{|c|}{$\begin{array}{l}\text { Zeit von der Ansteckung } \\
\text { bis zum Auftreten der ersten } \\
\text { Tabessymptome }\end{array}$} & & $\begin{array}{l}\text { Zabl } \\
\text { der } \\
\text { Fälle }\end{array}$ \\
\hline Bis 5 & Jahre & & & 14 \\
\hline von 5 & bis 10 & hren & & 40 \\
\hline$=10$ & $=15$ & $=$ & & 29 \\
\hline$=15$ & $=20$ & $=$ & & 22 \\
\hline$=20$ & $=25$ & $=$ & & 8 \\
\hline$=25$ & $=\mathbf{3 0}$ & $=$ & & $\left.3^{2}\right)$ \\
\hline
\end{tabular}

Wir sehen also, dass in den ersten 5 Jabren und nach Ablauf von 20 Jahren nach der Ansteckung Tabes selten auftritt, am häufigsten zwischen dem 5. bis 10. Jahre nach der Ansteckung; ziemlich oft jedoch auch von dem 10. bis 20. Jahre. Dies Ergebniss stimmt übrigens vollständig mit demjenigen anderer Autoren.

In Betreff des Alters der Kranken haben wir folgende Ergebnisse :

$$
\begin{aligned}
& \text { von } 20 \text { bis } 30 \text { Jahren ... } 29 \text { Kranke } \\
& =30=40=\ldots .103= \\
& =40=50=\ldots .89=
\end{aligned}
$$

1) Er drückt sich darüber aus, wie folgt: „Ueber die Thatsache des ätiologischen Zusammenhanges zwischen Tabes und Syphilis besteht unter den wirklich Sachverständigen keine Meinungsverschiedenheit mehr, wenn auch allerdings die Frage nach der Art und Weise dieses Zusammenhanges auch hente noch als ungelöst zu betrachten ist (l. c.).

2) In einem unserer Fălle trat Tabes angeblich nach 40 Jahren nach der Ansteckung auf. 


$$
\begin{aligned}
& \text { von } 50 \text { bis } 60 \text { Jahren ..... } 25 \text { Kranke } \\
& =60=70=\ldots .6= \\
& =70=80=\ldots \ldots 1 \text { ( } 72 \text { Jahre). }
\end{aligned}
$$

In ätiologischer Hinsicht ist auch das häufige Vorkommen der Tabes bei Aerzten sehr bemerkenswerth. Unter unseren 257 Fällen waren 12 Aerzte. Es beträgt dies 4,6 Proc., während das allgemeine Verhältniss von Aerzten zur gesammten Bevölkerung in Warschan nur ungefähr 0,2 Proc. beträgt (700 Aerzte auf 500000 Einwolnver). Natürlich lassen sich aus dem eben Gesagten keine bestimmten Folgerungen ziehen, denn es ist ja leicht möglich, dass wir auch bei anderen Krankheiten Analoges finden könnten.

II. Die Symptomatologie der Tabes dorsalis ist eine der best bearbeiteten Seiten der speciellen Pathologie, trotzdem giebt es noch verschiedene der Besprechung würdige Fragen, die wir auch weiter unten unter Zuziehung unseres Materiales beribren wollen.

In den meisten Fällen sind die Schmerzen das erste Symptom von Tabes. Le i $\mathrm{m} \mathrm{bac} \mathrm{h}^{1}$ ) giebt anf Grund von 900 Fällen aus der Klinik Erb's an, dass in 88,25 Proc. Fällen charakteristische lancinirende Schmerzen vorkommen. Soviel wir aus unserem Material schliessen können, ist diese Zahl eine ausnahmsweise grosse, soweit es sich wirklich um die charakteristischen Schmerzen handelt. Wir fanden nur bei der Hälfte unserer Kranken charakteristische und für die Erkennung der Krankheit bis zu einem gewissen Grade maassgebende Schmerzen. Auf die gesammte Zahl von 257 Fällen traten zwar Schmerzen in 225 , d. h. 87,5 Proc. ${ }^{2}$ ) auf, (also dasselbe Verhältniss wie bei Leimbach), von diesen aber waren sie nur in 112 Fällen mehr oder weniger charakteristisch. Uebrigens ist die Frage, welche Schmerzen man charakteristisch nennen kann, nicht leicht zu entscheiden, denn die Schmerzen bei Tabes sind sehr verschieden, und es ist sehr schwer, eine Definition anzugeben, welche allen oder wenigstens den meisten Fällen von Tabes entsprechen wïrde. Obgleich zweifellos, wie auch $G$ ow ers behauptet, Fälle von Tabes vorkommen, deren einziges Symptom Schmerzen sind (tabetische Neuralgie), ist es dennoch zweifelhaft, ob die Schmerzen je so pathognomonisch auftreten, dass ihr Vorhandensein die Diagnose von Tabes sicher stellt. Alle Kliniker, welche über ein grösseres Material verfügen, haben wohl Kranke angetroffen, deren Leiden Anfangs sich lediglich als

1) Statistisches zur Symptomatologie der Tabes dorsalis. Deutsche Zeitschrift für Nervenheilkande. 1895. Bd. VII. Heft 5 u. 6.

2) Zweifellos giebt es, wenn auch selten, Tabiker, welche Schmerzen hatten; es sind dies Fälle von schmerzloser Tabes - Tabes indolens. 
Neuralgie (am öftesten des $\mathbf{N}$. ischiadicus) oder Rheumatismus documentirte, erst später durch Hinzukommen anderer Symptome entpuppte sich die Tabes dorsalis, welche sich hinter der angeblichen Neuralgie verbarg. Die tabischen Schmerzen können jedoch einen solchen diagnostischen Werth beanspruchen, dass beim Vorbandensein noch eines wenn auch weniger wichtigen Symptomes, z. B. der Parästhesien, Blasenstörungen u. s. w. die Diagnose der Tabes dorsalis ziemlich sicher festgestellt werden kann. Die nächstfolgende Charakteristik der Schmerzen ist gänzlich dem uns zur Verfügung stehenden Material entnommen. Die für Tabes in obengenanntem Sinne cbarakteristischen Scbmerzen sind von verschiedener Intensität: oft so stark, dass sie den Schlaf des Kranken stören und seine einzige Klage ausmachen, oft jedoch erinnert sich der Leidende erst nach langem Ausfragen, dass bei ihm schwache Schmerzen vorbanden waren, die einen reissenden Charakter hatten; zuweilen bezeichnen die Kranken diese letzte Art als springenden Rheumatismus - diese Schmerzen, obgleich von schwacher Intensität, sind jedoch in anderer Hinsicht âusserst charakteristisch. Gewöhnlich erscheinen die Schmerzen ohne bestimmte Ursache, sind aber oft von der Veränderung der Witterung, überanstrengender Thätigkeit, Erkältung u. s. w. abhängig. Die Kranken bezeichnen sie als brennend, bohrend, einem elektrischen Schlage ähnlich; sie sind kurzdauernd, blitzartig, beschränken sich auf einen kleinen Raum und balten sich an einem bestimmten Punkte nur ziemlich kurze Zeit auf (manchmal nur einige Angenblicke, oft jedoch $1 / 2$ oder mehrere Stunden). Sie springen von einem Ort zum anderen und kebren zum ursprünglichen zurück. Gewöhnlich überfallen sie den Kranken in Attaquen, welche zusammen von einigen Stunden (auch weniger) bis zu einigen Tagen dauern, um dann auf einige Zeit zu verschwinden. Wäbrend des Bestehens der Schmerzen kommt oft Hyperästhesie der Haut an der schmerzhaften Stelle vor, welche auch nach dem Verschwinden der Schmerzen bleiben kann. In anderen Fällen erscheint im Gegentheil tactile Anästhesie, Analgesie oder Termanästhesie. An der schmerzhaften Stelle kann auch ein Errötben, sogar eine schnell verschwindende Schwellung vorkommen. Die Schmerzen können auch mit erhöhter localer Temperatur verbunden sein. Meistens sind die tabetischen Schmerzen an den unteren Extremitäten localisirt ${ }^{1}$ ), nicht allzu selten finden wir sie jedoch an den

1) Manchmal erscheinen sie im Verlaufe des N. ischiadicus, was leicht zu Irrthümern in der Diagnose führen kann; auch Gowers und Möbius erwähnen solche Fälle. Im Allgemeinen kommt die Localisation der Schmerzen bei Tabes im Verlaufe irgend eines Nerven sehr selten vor. 
oberen Extremitäten umưn Rampf, selten im Gesichte. Am contantesten scheinen folgende Eigenschaften zu sein: erstens, dass die Schmerzen, stark oder schwach, serienweise auftreten mit mehr oder weniger langen schmerzlosen Pausen; zweitens, dass sie sich nicht an einem bestimmten Orte halten, sondern von einem zum anderen überspringen; drittens, dass sie momentan von sehr kurzer Dauer sind. Wenn wir auf Schmerzen stossen, welche einige von den aufgezählten Eigenschaften besitzen, und daneben auch gewisse ätiologische (Syphilis) oder erbliche (Tabes bei anderen Mitgliedern der Familie) Momente vorkommen, dann haben wir einen berechtigten Verdacht, dass das Anfangsstadium der Tabes vorliegt; wenn solche Sehmerzen mit irgend einem der minderwichtigen tabetischen Symptome (Blasen- oder Geschlechtsstörungen, beschränkte Analgesie, Verdoppelung der Schmerzempfindung a. m. A.) verbunden sind, so wird die Diagnose sehr wahrscheinlich; wenn aber neben den tabetischen Schmerzen auch nur eins der wichtigen Symptome der Tabes auftritt, wie das Westphal'sche oder Argyll-Robertson'sche Symptom, dann ist die Diagnose vollständig sicher.

Alle anderen nicht sehr starken, oft als rheumatisch bezeichneten und überhanpt nicht die vorhin erwähnten Eigenschaften besitzenden Schmerzen müssen wir als nicht charakteristisch bezeichnen, und sie gewinnen erst in Verbindung mit anderen Symptomen den diagnostischen Werth.

III. Wenden wir uns jetzt zum zweiten Cardinalsymptome der Tabes, nämlich zum Westphal'schen Symptom. Leimbach fand in der sehon eitirten Arbeit dieses Zeichen (ein- oder beiderseitiges Fehlen oder dentliche Abschwächung der Patellar- oder Achillessehnereflexe) in 92 Proc. Fällen, Ungleichbeit zu beiden Seiten in 4,25 Proc Fällen, zusammen 96,25 Proc. In unseren Fällen haben wir folgende Ergebnisse (nur die Kniereflexe betreffend) gefunden:

Fehlen beider Knieretlexe . . . . in 178 Fällen $=68,4$ Proc.

$=$ eines Kniereflexes ..... = $=13=5=$

Abschwächung beider Kniereflexe . . = $12==4,6=$

Ungleichheit $=\quad \ldots=17==6,6=$

Im Allgemeinen also waren die Kniereflexe in 220 Fällen, was 85,6 Proc. der Gesammtzahl ausmacht, verändert.

Einiges Interesse erwecken die Fälle, aus welchen wir den Verlauf des Verschwindens der Kniereflexe ersehen können. In einem Falle schwanden die vorher notirten schwachen Kniereflexe schon nach einer Woche. In einem zweiten Falle waren beide Reflexe am 18. September 1886 vorhanden, am 17. Juni 1887 waren sie schwach und un- 
gleich, und am 8. Juli 1887 verseh mollständig. Im dritten Falle waren die Reflexe am 13. Mai 1888 ungleich, und am 19. Januar 1889 war an einer Seite der Kniereflex erloschen. Im vierten Falle waren die Reflexe am 21. Mai $1890 \mathrm{schwach}$, und am 29. Juni 1890 verschwanden sie gänzlich. Endlich im fünften Falle waren die Kniereflexe am 10. December 1887 ganz deutlich (Fehlen der Achillessehnenreflexe), am 25. December 1887 waren sie schwach, am 30. December 1887 liessen sie sich gar nicht hervorrufen. In einigen Fällen treffen wir auch neben der Anwesenbeit von Kniereflexen das Feblen der Achillessehnenreflexe. Am interessantesten sind jedoch die Fälle, in welchen die verschwundenen Reflexe wieder auftraten; diese Fälle wurden bereits anderswo beschrieben. ${ }^{1)}$ Weniger zahlreich wurde der Achillessehnenreflex berücksichtigt, dessen Verhalten nur in 116 Fällen angegeben wird; die betreffenden Fälle theilen wir in zwei Gruppen, je nachdem ob gleichzeitig der Patellarreflex normal oder verändert war.

Gruppe I. Fälle mit verändertem Patellarreflex bei gleichzeitigem beiderseitigem Fehlen des Achillessehnenreflexes $97 \mathrm{mal}$ einseitiges $==6=$ Ungleichheit des Achillessebnenreflexes ..... $2=$ Abschwächung des Achillessehnenreflexes .... $2=$

Gruppe II umfasst diejenigen Fälle, in welchen der Patellarreflex normal, dagegen

Achillessehnenreflex beiderseits fehlt ..... 5 mal

Ungleichheit des Reflexes............

IV. Zum Schluss führen wir eine Tabelle an, in welcher statistische Daten über andere Symptome, wie auch Complicationen der Tabes angegeben sind:

Fehlen der Pupillenreaction auf Licht einoder beiderseits . . . . . . in 141 Fällen $=54,8$ Proc.

Schwachheit der Pupillenreaction anf Licht beiderseits ......... $=52=-20,2=$

Ungleichheit der Pupillen ....... = $88==34,2=$

Lähmung der Augenmuskeln ...... $=56=21,7=$

Atrophie der Sehnerven ........ = $26=-10,1=$

Blasenstörungen .......... $=88==34,2=$

1) Goldfla m, Ueber die Ungleichheit der Kniephänomene bei Tabes dors. (Neurolog. Centralbl. 1888. Nr. 19 u. 20). Derselbe Autor: Ueber das Zurückerscheinen der Sehnenreflexe im Verlaufe der Tabes dorsalis (Kronika lekarska. 1890, polnisch). Diese Erscheinung erwähnt auch Gowers in seinem Handbuche der Nervenkrankheiten. 
Crises gastriques .......... in 15 Fällen

$=$ laryngées ............ zusammen

$=$ urinaires $\ldots \ldots \ldots \ldots=2\}=26=9,4$ Proc.

$=$ rectales $\ldots \ldots \ldots \ldots \ldots=1$

Hemiplegie ............ $=4$ Fällen

Paraplegie ............ $=2=$

Anaesthesia trigemini ......... = $2=$

Paralysis facialis........... = 1 Falle

$=$ ulnaris ........... = $=1=$

$=$ peronaei ............. = $1=$

$=$ vagoaccessorius ....... = $=1=$

Affectio N. acustici .......... = 2 Fällen

Ophthalmoplegia ext. et int. ...... = 1 Falle

Mal perforant du pied .......... = $=$

Zerreissung der Sehne des M. quadriceps $=1^{1}$ ) $=$

Ausfallen der Zähne........... = 2 Fällen ${ }^{2}$ )

Arthropathia tabetica ........ = $=$

Vitium cordis .......... = $=7=$

Mogigraphia ............ = $2=$

Epilepsia ............. = 1 Falle

Dementia paralytica ......... = $=1=$

Acute Psychose ............ = $1=$

Am Ende dieses Aufsatzes sei es mir gestattet, Herrn Dr. S. Gold flam für die Ueberlassung des Materiales, sowie für das Interesse an der Arbeit meinen herzlichsten Dank auszusprechen.

1) Der Kranke war behaftet mit Lues hereditaria et acquisita.

2) Bei einem von diesen Kranken fiel auch der Nagel der linken grossen Zehe ohne Ursache ganz schmerzios ab. 